www.jmscr.igmpublication.org

Impact Factor 5.84

Index Copernicus Value: 71.58

ISSN (e)-2347-176x ISSN (p) 2455-0450

crossref DOI: _https://dx.doi.org/10.18535/jmscr/v5i12.151

Journal Of Medical Science And Clinical Research

\title{
Treatment Outcome of Triple Negative Breast Cancer- A Retrospective Study from Regional Cancer Centre Kashmir
}

\author{
Authors \\ Wani Shahid Bashir ${ }^{1}$, Manirathinam Ramalingam ${ }^{2}$, Parray Muzafar Yousuf $f^{3}$, \\ Lone Mansoor Ul Haq ${ }^{4}$, Shah Saqib Ahmad ${ }^{5}$, Sofi Mushtaq Ahmad ${ }^{6}$, \\ Malik Tariq Rasool ${ }^{7}$ \\ ${ }^{1,4,5,6}$ Senior Resident, Radiation Oncology Skims \\ ${ }^{2}$ Fellow Radiation Oncology, Narayana Hrudayalaya Bangalore \\ ${ }^{3}$ Senior Resident, General Surgery Skims \\ ${ }^{7}$ Associate Professor Radiation Oncology Skims \\ Corresponding Author \\ Wani Shahid Bashir \\ Senior Resident, Radiation Oncology Skims
}

\section{Introduction}

Triple negative breast cancer (TNBC) is an uncommon subtype of breast cancer which lacks both hormone receptors (Estrogen receptor and Progestrone receptor) as well as Human epidermal growth factor receptor -2 . The lack of these receptors makes its prognosis challenging as neither hormonal therapy nor anti Her-2 targetted therapy serves as a treatment option. As a result TNBC accounts for a disproportionate number of breast cancer deaths, majority studies indicate a negative impact of triple negative phenotype on patient prognosis ${ }^{1,2,3}$. Importantly the prognostic effect of TNBC is independent of poor grade, nodal status, tumor size and treatment ${ }^{4}$. The aggressiveness of TNBC is further indicated by the fact that the peak recurrence occurs within the first 3 years after initial treatment of the disease with the majority of deaths occurring in the first 5 years ${ }^{5}$ and after diagnosis of metastatic disease a significantly shorter survival was observed in $\mathrm{TNBC}^{6,7}$. Conversely the risk for late recurrences i.e. beyond five years of diagnosis is decreased by $50 \%$ compared to hormone receptor positive disease $^{8}$. However differences between TNBC and non-TNBC regarding overall survival wears off at 10 years of follow up.

\section{Aim}

Aim of this study was to find out the treatment outcome with respect to sites of failure and survival analysis in triple negative breast cancer patients who were treated at our institute from 2008-2012.

\section{Methods}

The study was conducted in the Department of Radiation Oncology and Department of Medical Oncology SKIMS Srinagar. 
Inclusion criteria were breast cancer patients registered at hospital based cancer registry SKIMS from 2008-2012, who were documented as cases of TNBC by immunohistochemistry. Since data was collected retrospectively, no patient having Her2 2+ was subjected to FISH testing as all the patients had already completed treatment and were on follow up.

Exclusion criteria were patients with double malignancy and male breast cancer patients.

\section{Results}

First part of the study i.e. patient profile and characteristics are already published ${ }^{9}$. Here we will discuss the treatment and survival outcome of this patient cohort. Total number of patients in this study i.e. $\mathrm{n}$ is 94 .

Table 1. Distribution of cases according to the surgical procedure performed $(\mathrm{n}=94)$.

\begin{tabular}{|l|c|c|}
\hline Surgical procedure & $\mathrm{n}$ & Percent $(\%)$ \\
\hline Biopsy only & 2 & 2.1 \\
\hline BCS & 18 & 19.1 \\
\hline MRM & 74 & 77.8 \\
\hline
\end{tabular}

BCS: Breast conservative surgery, MRM: Modified radical mastectomy.

Most common surgical procedure performed in our cases was modified radical mastectomy. Among the 2 patients with biopsy only 1 didn't receive any further treatment and the other 1 received chemotherapy and radiation but was never disease free.

Table 2 Distribution of cases according to the chemotherapy regime received.

\begin{tabular}{|l|c|c|}
\hline Chemotherapy regime & $\mathrm{n}$ & Percent \\
\hline AC & 2 & 2.1 \\
\hline AC/T & 13 & 13.8 \\
\hline FAC & 12 & 12.7 \\
\hline FAC/T & 3 & 3.2 \\
\hline FEC & 35 & 37.2 \\
\hline FEC/T & 13 & 13.8 \\
\hline GEMCIS & 6 & 6.4 \\
\hline Others & 10 & 10.6 \\
\hline
\end{tabular}

A:Adriamycin, C:Cyclophosphamide, T:Paclitaxel, F:5-FU, E:Epirubicin, GEMCIS: Gemcitabine+Cisplatin. Most common chemo regimes used in our study were FEC, FEC/T and AC/T.
Table 3 Distribution of cases according to radiotherapy received.

\begin{tabular}{|l|c|c|}
\hline Radiotherapy recieved & $\mathrm{n}$ & Percent \\
\hline yes & 74 & 78.7 \\
\hline no & 20 & 21.2 \\
\hline
\end{tabular}

$78.7 \%$ of our cases received radiotherapy as a part of multimodality treatment.

Table 4 Distribution of cases according to locoregional failure.

\begin{tabular}{|l|c|c|}
\hline Loco-regional failure & $\mathrm{n}$ & Percent \\
\hline Yes & 5 & 5.3 \\
\hline No & 89 & 94.6 \\
\hline
\end{tabular}

$5.3 \%$ of the patients experienced loco-regional failure.

Table 5 Distribution of cases according to Bone metastasis.

\begin{tabular}{|l|c|c|}
\hline Bone metastasis & $\mathrm{n}$ & Percent \\
\hline Yes & 8 & 8.5 \\
\hline No & 86 & 91.5 \\
\hline
\end{tabular}

$8.5 \%$ of our cases developed bone metastasis. Out of these 8 patients, 7 developed metastasis in less than 1 year of diagnosis.

Table 6 Distribution of cases according to Visceral metastasis.

\begin{tabular}{|l|c|c|}
\hline Visceral metastasis & $\mathrm{n}$ & Percent \\
\hline Yes & 12 & 12.6 \\
\hline No & 82 & 87.2 \\
\hline
\end{tabular}

$12.6 \%$ of the cases developed distant visceral metastasis. Most common site of visceral metastasis in this study was Brain in $6.3 \%$ cases.

Table 7 Distribution of cases according to their survival analysis.

\begin{tabular}{|l|c|c|}
\hline Survival & Range $(\mathrm{m})$ & Mean $(\mathrm{m})$ \\
\hline Disease-free survival & $0-52$ & 13.6 \\
\hline Overall survival & $2-60$ & 22.4 \\
\hline
\end{tabular}

m:months. Mean follow up was 14.2 months.6 month overall survival in this study was $91.5 \%$. likewise 1,2 and 3 year overall survival in our patients was $70.9,57.3$ and 60 percent respectively.

\section{Discussion}

Triple negative breast cancer is considered bad prognostic phenotype of breast cancer. Primary basis for its bad prognosis is the absence of any 
receptors against which hormonal or other targeted agents are available. Triple negative breast cancer is closely related to basal like breast cancer (BLBC) and like BLBC triple negative breast cancer is also associated with BRCA mutations. Though theoretically BRCA mutation positivity makes TNBC especially susceptible to alkylating agents like cisplatin but same is yet to be proven in studies. As such TNBC in our centre is treated as a high risk breast cancer usually with anthracycline followed by a taxane based chemotherapy. Surgical management and Radiation treatments remain the same as for other subtypes of breast cancer with respect to stage of disease.

In this study majority of our patients underwent modified radical mastectomy as shown in Table 1. All our patients had histology of infiltrating ductal carcinoma and most common stage at presentation was Stage II(60\%) and Stage III $(37 \%)^{9}$.

All the patients in this study received chemotherapy. Most common regimes used were FEC in $37.2 \%$ followed by FEC/T and AC/T both in $13.8 \%$ respectively as depicted in Table 2 . Most of our patients i.e $79 \%$ received radiation to local and regional sites while $21 \%$ didn't. Out of these $21 \%$ cases, $6 \%$ had no indication of adjuvant radiotherapy, $12.7 \%$ were lost to follow up after chemotherapy and $2.1 \%$ never achieved remission and were alive with disease.

Mean timing of starting chemotherapy after surgery in our patients was 1.26 months and mean timing of radiotherapy after surgery and chemotherapy was 7.43 and 1.8 months respectively.

Locoregional failure was observed in $5.3 \%$ of the cases as depicted in Table 4. Mean disease free survival in this subset of patients was 3.2 months. Among these 5 patients, 1 had underwent biopsy only, 2 patients underwent breast conserving surgery, 1 patient received chemotherapy only and the other 1 received chemotherapy and radiation. Distant metastasis was observed in $21 \%$ of the cases out of which bone metastasis was seen in $8.5 \%$ of the cases as depicted in Table 5 and visceral metastasis was seen in $12.6 \%$ of the cases. Most common site of visceral metastasis was brain followed by liver. At an average among the patients developing distant metastasis, brain metastasis developed in $1^{\text {st }}$, liver in $2^{\text {nd }}$ and lung metastasis in $3^{\text {rd }}$ year of diagnosis.

$\mathrm{R}$ Dent et $\mathrm{al}^{6}$ found that $13 \%$ of the patients having triple negative breast cancer recur locally as compared to $12 \%$ in the other types of breast cancer but a higher proportion of patients having triple negative breast cancer i.e. $34 \%$ experienced distant metastasis when compared to other types of breast cancer i.e. $20.4 \%$. These findings are not in completed concordance with our observations.

Lin NU et al ${ }^{10}$ and Heitz et al ${ }^{11}$ observed that brain metastasis occurs in 6-46\% of triple negative breast cancer patients undergoing metastatic spread of disease which is again not in complete concordance with our observations.

Mean disease free survival in our study was 13.6 months, mean overall survival was 22.4 months and mean follow up period was 14.2 months as illustrated in Table 7. Majority of our patients were disease free at the last follow up but follow up period was too short to determine exact disease free survival. Some patients had recently completed their treatment but didn't contribute to disease free survival because of very short follow up. Longer follow up is needed to know exact survival data in this patient cohort.

Overall survival at 6 months, 1,2 and 3 years in this study was 91.5, 70.9, 57.3 and $60.0 \%$ respectively. Leidke et $\mathrm{al}^{12}$ observed that overall survival in triple negative breast cancer at 1 and 3 years was $90 \%$ and $74 \%$ while comparing it to non TNBC in which it was $97 \%$ and $89 \%$ respectively.

Dawood et $\mathrm{al}^{13}$ observed that 2 year overall survival in triple negative breast cancer patients was $85.9 \%$.

In our study 1, 2 and 3 year overall survival was less when compared to the above mentioned studies which could be due to small subsets of patients as 1 year survival was calculated in 86 
patients, 2 and 3 year survival was calculated in 61 and 35 patients only respectively.

Comparing the outcome analysis of our patient population with the published data, some of the trends were similar i.e. brain and liver were most common sites of visceral metastasis though incidence was at the lower end. This could be due to relatively short follow up and small patient cohort in our study.

Considering the pattern of failure, aggressive follow up with HPE and imaging is probably a preferable option to detect the recurrent lesions early but a long term follow up study is needed to clear these differences between TNBC and non TNBC.

\section{Conclusion}

Triple negative breast cancer is an aggressive subtype of breast cancer which tends to recur early, more commonly at distant sites especially brain and bone which is what we observed in this study.

Disease free and overall survival in this study was 13.6 and 22.4 months respectively but mean follow up period was only 14.15 months.

1,2 and 3 year survival in this study was $70.9 \%$, $57.3 \%$ and $60 \%$ respectively which is on the lower end of published data but this difference could be attributed to relatively short follow up in our study.

Larger study size and longer follow up periods are required to clear these differences.

\section{References}

1. Carey LA, Dees EC, Sawyer L, et al: The triple negative paradox: Primary tumor chemosensitivity of breast cancer subtypes. Clin Cancer Res 13::2329,20072334.

2. Sorlie T1, Tibshirani R, Parker J et al. Repeated observation of breast tumor subtypes in independent gene expression data sets. Proc Natl Acad Sci U S A. 2003 Jul 8;100(14):8418-23.
3. Abd El-Rehim DM, Ball G, Pinder SE, et al. High-throughput protein expression analysis using tissue microarray technology of a large well-characterised series identifies biologically distinct classes of breast cancer confirming recent cDNA expression analyses. Int J Cancer 2005;116:340-50.

4. Hugh J, Hanson J, Cheang MC, et al. Breast cancer subtypes and response to docetaxel in node-positive breast cancer: use of an immunohistochemical definition in the BCIRG 001 Trial, J Clin Oncol , 2009, vol. 27 (pg. 1168-1176).

5. Tischkowitz M, Brunet JS, Begin LR et al. Use of immunohistochemical markers can refine prognosis in triple negative breast cancer. BMC Cancer 2007; 7; 134.

6. Dent R, Trudeau M, Pritchard KI, et al: Triple-negative breast cancer: Clinical features and patterns of recurrence. Clin Cancer Res 13::4429,2007-4434.

7. Harris LN, Broadwater G, Lin $\mathrm{NU}$ et al. Molecular subtypes of breast cancer in relation to paclitaxel response and outcomes in women with metastatic disease: results from CALGB 9342. Breast Cancer Res.2006; 8; R66.

8. Nofech-Mozes S, Trudeau M, Kahn H, et al. Patterns of recurrence in the basal and non-basal subtypes of triple-negative breast cancers, Breast Cancer Res Treat , 2009, vol. 118 (pg. 131-137).

9. Wani Shahid Bashir, Shah Saqib Ahmad, Lone Mansoor et al. Incidence and clinical profile of Triple negative breast cancer in Kashmir.jmscr Volume 05 Issue 04 April 2017:20746-20749.

10. Lin NU, Claus E, Sohl J, et al. Sites of distant recurrence and clinical outcomes in patients with metastatic triple-negative breast cancer, Cancer, 2008, vol. 113 (pg. 2638-2645). 
11. Heitz F, Harter P, Traut A, et al. Cerebral metastases $(\mathrm{CM})$ in breast cancer $(\mathrm{BC})$ with focus on triple-negative tumors, $\mathrm{J}$ Clin Oncol (Meeting Abstracts) , 2008, vol. 26 (Abstr 1010).

12. Liedtke C, Mazouni C, Hess KR, et al. Response to neoadjuvant therapy and longterm survival in patients with triplenegative breast cancer, J Clin Oncol, 2008, vol. 26 (pg. 1275-1281).

13. Dawood S, Broglio K, Esteva FJ, et al. Survival among women with triple receptor-negative breast cancer and brain metastases, Ann Oncol, 2009, vol. 20 (pg. 621-627). 